\title{
Triple fixed point theorems on FLM algebras
}

\author{
Abdolrahman Razani* and Hasan Hosseinzadeh
}

"Correspondence: razani@ipm.ir Department of Mathematics, Karaj Branch, Islamic Azad University, Karaj, Iran

\begin{abstract}
This paper considers tripled fixed point theorems on unital without of order semi-simple fundamental locally multiplicative topological algebras (abbreviated by FLM algebras).

MSC: $46 \mathrm{H}$

Keywords: tripled fixed point; fundamental topological algebras; FLM algebras; holomorphic function; semi-simple algebras; without of order
\end{abstract}

\section{Introduction}

Ansari in [1] introduced the notion of fundamental topological spaces and algebras and proved Cohen's factorization theorem for these algebras. A topological linear space $\mathcal{A}$ is said to be fundamental if there exists $b>1$ such that for every sequence $\left(x_{n}\right)$ of $\mathcal{A}$, the convergence of $b^{n}\left(x_{n}-x_{n-1}\right)$ to zero in $\mathcal{A}$ implies that $\left(x_{n}\right)$ is Cauchy. A fundamental topological algebra is an algebra whose underlying topological linear space is fundamental.

A fundamental topological algebra is called locally multiplicative if there exists a neighborhood $U_{0}$ of zero such that for every neighborhood $V$ of zero, the sufficiently large powers of $U_{0}$ lie in $V$. The fundamental locally multiplicative topological algebras (FLM) were introduced by Ansari in [2]. Some celebrated theorems in Banach algebras were generalized to FLM algebras in [3], and authors investigated some fixed points theorems for holomorphic functions on these algebras (see Theorems 3.5, 3.6 and 3.7 of [3]).

An algebra $\mathcal{A}$ is called without of order if for every $a, b \in \mathcal{A}, a b=0$, then $a=0$ or $b=0$. In [4], Bhaskar and Lakshmikantham introduced the notions of a mixed monotone mapping and a coupled fixed point, proved some coupled fixed point theorems for the mixed monotone mapping and discussed the existence and uniqueness of a solution for a periodic boundary value problem. Also, Samet and Vetro studied a coupled fixed point of $N$-order in [5]. There are many works on a coupled fixed point of contraction, weak contraction and generalized contraction mappings on various metric spaces such as [6-9].

Let $\mathcal{A}$ be a metric space and let $F: \mathcal{A} \times \mathcal{A} \times \mathcal{A} \longrightarrow \mathcal{A}$ be a function. An element $(x, y, z) \in$ $\mathcal{A} \times \mathcal{A} \times \mathcal{A}$ is said to be a tripled fixed point of the mapping $F$ if $F(x, y, z)=F(x, z, y)=x$, $F(y, x, z)=F(y, z, x)=y$ and $F(z, x, y)=F(z, y, x)=z$. Tripled fixed point theorems in partially ordered metric spaces were studied by Berinde and Borcut in [10], and this concept was considered by Aydi et al. for weak compatible mappings in abstract metric spaces [11].

In this paper, at first (Section 2) we obtain some basic results for FLM algebras, and next we consider tripled fixed point theorems on FLM algebras. 


\section{Some results on FLM algebras}

By $\Omega_{\mathcal{A}}$ we mean the set of all elements $a \in \mathcal{A}$ such that $\rho_{\mathcal{A}}(a)<1$, where $\rho_{\mathcal{A}}(a)$ is the spectral radius of $a \in \mathcal{A}$. We denote the center of topological algebra $\mathcal{A}$ by $Z(\mathcal{A})$ such that

$$
Z(\mathcal{A})=\{a \in \mathcal{A}: a x=x a \text { for all } x \in \mathcal{A}\}
$$

Definition 2.1 Let $(\mathcal{A}, d)$ be a metrizable topological algebra. We say $\mathcal{A}$ is a submultiplicatively metrizable topological algebra if

$$
d(0, x y z) \leq d(0, x) d(0, y) d(0, z) \quad \text { and } \quad d(0, \lambda x)<|\lambda| d(0, x)
$$

for each $x, y, z \in \mathcal{A}$ and $\lambda \in \mathbb{C}$. For abbreviation, we denote $d_{\mathcal{A}}(0, x)$ by $D_{\mathcal{A}}(x)$ for any $x \in \mathcal{A}$.

Let $\mathcal{A}, \mathcal{B}$ and $\mathcal{C}$ be metric spaces with meters $d_{\mathcal{A}}, d_{\mathcal{B}}$ and $d_{\mathcal{C}}$, respectively. Then $\mathcal{A} \times \mathcal{B} \times \mathcal{C}$ becomes a metric space with the following meter:

$$
d\left(\left(a_{1}, b_{1}, c_{1}\right),\left(a_{2}, b_{2}, c_{2}\right)\right)=d_{\mathcal{A}}\left(a_{1}, a_{2}\right)+d_{\mathcal{B}}\left(b_{1}, b_{2}\right)+d_{\mathcal{C}}\left(c_{1}, c_{2}\right)
$$

for every $a_{1}, a_{2} \in \mathcal{A}, b_{1}, b_{2} \in \mathcal{B}$ and $c_{1}, c_{2} \in \mathcal{C}$. When $\mathcal{A}, \mathcal{B}$ and $\mathcal{C}$ are algebras, then by the usual point-wise definitions for addition, scalar multiplication and product, $\mathcal{A} \times \mathcal{B} \times \mathcal{C}$ becomes an algebra.

Proposition 2.2 Let $\mathcal{A}, \mathcal{B}$ and $\mathcal{C}$ be complete metrizable FLM algebras with submultiplicative meters $d_{\mathcal{A}}, d_{\mathcal{B}}$ and $d_{\mathcal{C}}$, respectively. Then $\mathcal{A} \times \mathcal{B} \times \mathcal{C}$ is a complete metrizable FLM algebra with a submultiplicative meter d.

Proof Let $\mathcal{A}, \mathcal{B}$ and $\mathcal{C}$ be FLM algebras with meters $d_{\mathcal{A}}, d_{\mathcal{B}}$ and $d_{\mathcal{C}}$, respectively. By the definition of FLM algebras, obviously, $\mathcal{A} \times \mathcal{B} \times \mathcal{C}$ is a complete metrizable FLM algebra with a meter $d$ (the meter defined in (2.1)). For submultiplicativity, we have

$$
\begin{aligned}
d( & \left.(0,0,0),\left(a_{1} a_{2}, b_{1} b_{2}, c_{1} c_{2}\right)\right) \\
= & d_{\mathcal{A}}\left(0, a_{1} a_{2}\right)+d_{\mathcal{B}}\left(0, b_{1} b_{2}\right)+d_{\mathcal{C}}\left(0, c_{1} c_{2}\right) \\
\leq & d_{\mathcal{A}}\left(0, a_{1}\right) d_{\mathcal{A}}\left(0, a_{2}\right)+d_{\mathcal{B}}\left(0, b_{1}\right) d_{\mathcal{B}}\left(0, b_{2}\right)+d_{\mathcal{C}}\left(0, c_{1}\right) d_{\mathcal{C}}\left(0, c_{2}\right) \\
\leq & d_{\mathcal{A}}\left(0, a_{1}\right) d_{\mathcal{A}}\left(0, a_{2}\right)+d_{\mathcal{A}}\left(0, a_{1}\right) d_{\mathcal{B}}\left(0, b_{2}\right)+d_{\mathcal{A}}\left(0, a_{1}\right) d_{\mathcal{C}}\left(0, c_{2}\right) \\
& +d_{\mathcal{B}}\left(0, b_{1}\right) d_{\mathcal{A}}\left(0, a_{2}\right)+d_{\mathcal{B}}\left(0, b_{1}\right) d_{\mathcal{B}}\left(0, b_{2}\right) \\
& +d_{\mathcal{B}}\left(0, b_{1}\right) d_{\mathcal{C}}\left(0, c_{2}\right)+d_{\mathcal{C}}\left(0, c_{1}\right) d_{\mathcal{A}}\left(0, a_{2}\right) \\
& +d_{\mathcal{C}}\left(0, c_{1}\right) d_{\mathcal{B}}\left(0, b_{2}\right)+d_{\mathcal{C}}\left(0, c_{1}\right) d_{\mathcal{C}}\left(0, c_{2}\right) \\
= & d\left((0,0,0),\left(a_{1}, b_{1}, c_{1}\right)\right) d\left((0,0,0),\left(a_{2}, b_{2}, c_{2}\right)\right)
\end{aligned}
$$

for every $a_{1}, a_{2} \in \mathcal{A}, b_{1}, b_{2} \in \mathcal{B}$ and $c_{1}, c_{2} \in \mathcal{C}$. Also,

$$
\begin{aligned}
d((0,0,0),(\lambda a, \lambda b, \lambda c)) & =d_{\mathcal{A}}(0, \lambda a)+d_{\mathcal{B}}(0, \lambda b)+d_{\mathcal{C}}(0, \lambda c) \\
& <|\lambda| d_{\mathcal{A}}(0, a)+|\lambda| d_{\mathcal{B}}(0, b)+|\lambda| d_{\mathcal{C}}(0, c)
\end{aligned}
$$




$$
\begin{aligned}
& =|\lambda|\left(d_{\mathcal{A}}(0, a)+d_{\mathcal{B}}(0, b)+d_{\mathcal{C}}(0, c)\right) \\
& =|\lambda|(d((0,0,0),(a, b, c))) .
\end{aligned}
$$

Therefore, (2.2) and (2.3) show that $d$ is submultiplicative.

Similar to Definition 2.1, we write $D_{\mathcal{A} \times \mathcal{B} \times \mathcal{C}}(a, b, c)$ as an abbreviation for $d((0,0,0)$, $(a, b, c))$. We recall the following theorem from [3].

Theorem 2.3 [3, Theorem 3.3] Let $\mathcal{A}$ be a complete metrizable FLM algebra with a submultiplicative meter $d_{\mathcal{A}}$. Then $\rho(x)=\lim _{n \rightarrow \infty} D_{\mathcal{A}}\left(x^{n}\right)^{1 / n}$.

Lemma 2.4 Let $\mathcal{A}, \mathcal{B}$ and $\mathcal{C}$ be complete metrizable FLM algebras with submultiplicative meters $d_{\mathcal{A}}, d_{\mathcal{B}}$ and $d_{\mathcal{C}}$, respectively. Then

$$
\rho(x, y, z) \leq \rho_{\mathcal{A}}(x)+\rho_{\mathcal{B}}(y)+\rho_{\mathcal{C}}(z)
$$

for any element $(x, y, z) \in \mathcal{A} \times \mathcal{B} \times \mathcal{C}$.

Proof For given $a \in \mathcal{A}, b \in \mathcal{B}$ and $c \in \mathcal{C}$, we have $\rho_{\mathcal{A}}(a)=\lim _{n \rightarrow \infty} D_{\mathcal{A}}\left(a^{n}\right)^{1 / n}, \rho_{\mathcal{B}}(b)=$ $\lim _{n \rightarrow \infty} D_{\mathcal{B}}\left(b^{n}\right)^{1 / n}$ and $\rho_{\mathcal{C}}(c)=\lim _{n \rightarrow \infty} D_{\mathcal{C}}\left(c^{n}\right)^{1 / n}$ (Theorem 2.3). From Proposition 2.2, it follows that $\mathcal{A} \times \mathcal{B} \times \mathcal{C}$ is a complete metrizable FLM algebra with a submultiplicative meter $d$. Then again, Theorem 2.3 implies that

$$
\begin{aligned}
\rho(x, y, z) & =\lim _{n \rightarrow \infty} D_{\mathcal{A} \times \mathcal{B} \times \mathcal{C}}\left((x, y, z)^{n}\right)^{\frac{1}{n}}=\lim _{n \rightarrow \infty} D_{\mathcal{A} \times \mathcal{B} \times \mathcal{C}}\left(\left(x^{n}, y^{n}, z^{n}\right)\right)^{\frac{1}{n}} \\
& =\lim _{n \rightarrow \infty}\left(D_{\mathcal{A}}\left(x^{n}\right)+D_{\mathcal{B}}\left(y^{n}\right)+D_{\mathcal{C}}\left(z^{n}\right)\right)^{\frac{1}{n}} \\
& \leq \lim _{n \rightarrow \infty} D_{\mathcal{A}}\left(x^{n}\right)^{\frac{1}{n}}+\lim _{n \rightarrow \infty} D_{\mathcal{B}}\left(y^{n}\right)^{\frac{1}{n}}+\lim _{n \rightarrow \infty} D_{\mathcal{C}}\left(z^{n}\right)^{\frac{1}{n}} \\
& =\rho_{\mathcal{A}}(x)+\rho_{\mathcal{B}}(y)+\rho_{\mathcal{C}}(z)
\end{aligned}
$$

for every $x \in \mathcal{A}, y \in \mathcal{B}$ and $z \in \mathcal{C}$.

Similar to $\Omega_{\mathcal{A}}$ and $Z(\mathcal{A})$, we define these sets for $\mathcal{A} \times \mathcal{A} \times \mathcal{A}$ as follows:

$$
\Omega_{\mathcal{A} \times \mathcal{A} \times \mathcal{A}}=\{(x, y, z) \in \mathcal{A} \times \mathcal{A} \times \mathcal{A}: \rho(x, y, z)<1\}
$$

and

$$
\begin{aligned}
Z(\mathcal{A} \times \mathcal{A} \times \mathcal{A})= & \{(x, y, z) \in \mathcal{A} \times \mathcal{A} \times \mathcal{A}:(x, y, z)(a, b, c)=(a, b, c)(x, y, z), \\
& \text { for every } a, b, c \in \mathcal{A}\} \\
= & \{(x, y, z) \in \mathcal{A} \times \mathcal{A} \times \mathcal{A}:(x a, y b, z c)=(a x, b y, c z), \\
& \text { for every } a, b, c \in \mathcal{A}\} .
\end{aligned}
$$

Clearly, if $(x, y, z) \in Z(\mathcal{A} \times \mathcal{A} \times \mathcal{A})$, then $x, y, z \in Z(\mathcal{A})$ and $Z(\mathcal{A}) \subseteq Z(\mathcal{A} \times \mathcal{A} \times \mathcal{A})$. Also,

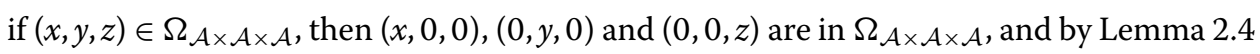
and its proof, we have $x, y, z \in \Omega_{\mathcal{A}}$. 
Let $E(\mathcal{A})$ be the set of all elements $x \in \mathcal{A}$ for which $E(x)=\sum_{n=1}^{\infty} \frac{x^{n}}{n !}$ can be defined. If $\mathcal{A}$ is a complete metrizable FLM algebra, then $E(\mathcal{A})=\mathcal{A}$ ([12, Theorem 5.4]). Therefore, in the light of Theorem 5.4 of [12] and Proposition 2.2, we have the following theorem.

Theorem 2.5 Let $\mathcal{A}$ be a complete metrizable FLM algebra, then $E(\mathcal{A} \times \mathcal{A} \times \mathcal{A})=\mathcal{A} \times$ $\mathcal{A} \times \mathcal{A}$.

\section{Tripled fixed point theorems}

In this section, we consider some results about tripled fixed point theorems on unital complete semi-simple metrizable FLM algebras, and we extend these results to Banach algebras. By id ${ }_{\mathcal{A}}$, we mean the identity map on $\mathcal{A}$.

Theorem 3.1 Let $\mathcal{A}$ be a unital without of order complete semi-simple metrizable FLM algebra with a submultiplicative meter $d_{\mathcal{A}}$. If $F: \Omega_{\mathcal{A} \times \mathcal{A} \times \mathcal{A}} \subseteq \mathcal{A} \times \mathcal{A} \times \mathcal{A} \rightarrow \Omega_{\mathcal{A}}$ is a holomorphic map that satisfies the conditions $F(0,0,0)=0, \frac{\partial F}{\partial x}(0,0,0)=\operatorname{id}_{\mathcal{A}}, \frac{\partial F}{\partial y}(0,0,0)=$ $0, \frac{\partial F}{\partial z}(0,0,0)=0, \frac{\partial^{2} F}{\partial x^{i} \partial j^{j} \partial z^{k}}(0,0,0)=0$, where $i+j+k=2, i, j, k=0,1,2$, and $\frac{\partial^{3} F}{\partial x^{i} \partial y^{j} j z^{k}}(0,0,0)=$ 0 , where $i+j+k=3, i, j, k=0,1,2,3$, then every $(a, b, c) \in \Omega_{\mathcal{A} \times \mathcal{A} \times \mathcal{A}} \cap Z(\mathcal{A} \times \mathcal{A} \times \mathcal{A})$ is a tripled fixed point for $F$.

Proof Fix $(a, b, c) \in \Omega_{\mathcal{A} \times \mathcal{A} \times \mathcal{A}} \cap Z(\mathcal{A} \times \mathcal{A} \times \mathcal{A})$ and consider the map $f: \mathbb{C} \times \mathbb{C} \times \mathbb{C} \longrightarrow \Omega_{\mathcal{A}}$ with $f(\alpha, \beta, \gamma)=F(\alpha a, \beta b, \gamma c)$. Clearly, $f$ is a holomorphic function on

$$
\begin{aligned}
& \left\{(\alpha, \beta, \gamma) \in \mathbb{C} \times \mathbb{C} \times \mathbb{C}: \frac{|\theta|}{3}<\frac{1}{\rho(a, b, c)},|\theta|=\min \{|\alpha|,|\beta|,|\gamma|\},\right. \\
& \left.\rho_{\mathcal{A}}(a)<\frac{1}{|\alpha|}, \rho_{\mathcal{B}}(b)<\frac{1}{|\beta|}, \rho_{\mathcal{C}}(c)<\frac{1}{|\gamma|}\right\} .
\end{aligned}
$$

Since $F(0,0,0)=0, \frac{\partial F}{\partial x}(0,0,0)=\operatorname{id}_{\mathcal{A}}, \frac{\partial F}{\partial y}(0,0,0)=0, \frac{\partial F}{\partial z}(0,0,0)=0, \frac{\partial^{2} F}{\partial x^{i} \partial y^{j} \partial z^{k}}(0,0,0)=0$, where $i+j+k=2, i, j, k=0,1,2$, and $\frac{\partial^{3} F}{\partial x^{i} \partial y^{j} \partial z^{k}}(0,0,0)=0$, where $i+j+k=3, i, j, k=0,1,2,3$, then $F$ has a Taylor expansion about $(0,0,0)$ :

$$
\begin{aligned}
F(x, y, z) & =\sum_{i=0}^{\infty} \sum_{j=0}^{\infty} \sum_{k=0}^{\infty} \frac{x^{i} y^{j} z^{k}}{i ! j ! k !}\left(\frac{\partial^{i+j+k} F}{\partial x^{i} \partial y^{j} \partial z^{k}}\right)(0,0,0) \\
& =x+\sum_{k=0}^{\infty} \frac{1}{k !} \sum_{j=0}^{k}\left(\begin{array}{c}
k \\
j
\end{array}\right) \sum_{i=0}^{j}\left(\begin{array}{c}
k-j \\
i
\end{array}\right) x^{i} y^{j} z^{k-i-j}\left(\frac{\partial^{k} F}{\partial x^{i} \partial y^{j} \partial z^{k-i-j}}\right)(0,0,0)
\end{aligned}
$$

for every $(x, y, z) \in \Omega_{\mathcal{A} \times \mathcal{A} \times \mathcal{A}} \cap Z(\mathcal{A} \times \mathcal{A} \times \mathcal{A})$. Therefore,

$$
\begin{aligned}
F(\alpha a, \beta b, \gamma c)= & \alpha a+\sum_{k=4}^{\infty} \frac{1}{k !} \sum_{j=0}^{k}\left(\begin{array}{c}
k \\
j
\end{array}\right) \sum_{i=0}^{j}\left(\begin{array}{c}
k-j \\
i
\end{array}\right) \alpha^{i} a^{i} \beta^{j} b^{j} \gamma^{k-i-j} c^{k-i-j} \\
& \times\left(\frac{\partial^{k} F}{\partial x^{i} \partial y^{j} \partial z^{k-i-j}}\right)(0,0,0) .
\end{aligned}
$$

We claim that

$$
\sum_{j=0}^{k}\left(\begin{array}{c}
k \\
j
\end{array}\right) \sum_{i=0}^{j}\left(\begin{array}{c}
k-j \\
i
\end{array}\right) \alpha^{i} a^{i} \beta^{j} b^{j} \gamma^{k-i-j} c^{k-i-j}\left(\frac{\partial^{k} F}{\partial x^{i} \partial y^{j} \partial z^{k-i-j}}\right)(0,0,0)
$$


is zero for every $k \geq 4$. Assume towards a contradiction that there exists $k \geq 4$ such that (3.2) is non-zero. Let $l \geq 4$ be an integer such that

$$
\sum_{j=0}^{k}\left(\begin{array}{l}
l \\
j
\end{array}\right) \sum_{i=0}^{j}\left(\begin{array}{c}
l-j \\
i
\end{array}\right) \alpha^{i} a^{i} \beta^{j} b^{j} \gamma^{l-i-j} c^{l-i-j}\left(\frac{\partial^{k} F}{\partial x^{i} \partial y^{j} \partial z^{l-i-j}}\right)(0,0,0) \neq 0
$$

Suppose that $q$ is an element of $\mathcal{A}$ such that $\rho_{\mathcal{A}}(q)=0$. Now, we consider the following five cases:

(1) $i=l, j=0$,

(2) $i=0, j=l$,

(3) $i+j=l$,

(4) $1 \leq i+j<l$,

(5) $i=j=0$.

Case (1). In this case, we have $\alpha^{l} a^{l} \frac{\partial^{l} F}{\partial x^{l}}(0,0,0) \neq 0$. Let $n \geq 1$, by (3.1) and (3.3), we have

$$
\begin{aligned}
F\left(n^{\frac{1}{l}} \alpha a+n \alpha^{l} q, \beta b, \gamma c\right)= & n^{\frac{1}{l}} \alpha a+n \alpha^{l} q+\frac{1}{l !}\left(n^{\frac{1}{l}} \alpha a+n \alpha^{l} q\right) \frac{\partial^{l} F}{\partial x^{l}}(0,0,0) \\
= & n^{\frac{1}{l}} \alpha a+n \alpha^{l} q+\frac{1}{l !}\left(n^{l} \alpha^{l^{2}} q^{l}+\ln ^{\frac{1}{l}} \alpha a n^{l-1} \alpha^{l(l-1)} q^{l-1}\right. \\
& \left.+\cdots+n \alpha^{l} a^{l}\right) \frac{\partial^{l} F}{\partial x^{l}}(0,0,0) \\
= & n^{\frac{1}{l}} \alpha a+n \alpha^{l}\left(q+\frac{1}{l !} a^{l} \frac{\partial^{l} F}{\partial x^{l}}(0,0,0)\right)+P(\alpha) \frac{\partial^{l} F}{\partial x^{l}}(0,0,0) .
\end{aligned}
$$

In (3.4), by $P(\alpha)$, we mean the remaining part of $\left(n^{\frac{1}{l}} \alpha a+n \alpha^{k} q\right)^{k}$. Since $a \in Z(\mathcal{A})$, therefore $a q=q a$. Then Lemma 2.4 and Lemma 3.6 of [3] imply

$$
\begin{aligned}
\rho\left(n^{\frac{1}{l}} \alpha a+n \alpha^{l} q, \beta b, \gamma c\right) & \leq \rho_{\mathcal{A}}\left(n^{\frac{1}{l}} \alpha a+n \alpha^{l} q\right)+\rho_{\mathcal{A}}(\beta b)+\rho_{\mathcal{A}}(\gamma c) \\
& <n^{\frac{1}{l}}|\alpha| \rho_{\mathcal{A}}(a)+|\beta| \rho_{\mathcal{A}}(b)+|\gamma| \rho_{\mathcal{A}}(c) \\
& <\mu\left(\rho_{\mathcal{A}}(a)+\rho_{\mathcal{A}}(b)+\rho_{\mathcal{A}}(c)\right),
\end{aligned}
$$

where $\mu=\max \left\{n^{\frac{1}{7}}|\alpha|,|\beta|,|\gamma|\right\}$. Now, we define a holomorphic function $H$ from $\{\alpha \in \mathbb{C}$ : $\left.0<|\alpha|<\frac{1}{\rho(a, b, c)}\right\}$ into $\mathcal{A}$ as follows:

$$
H(\alpha)=\frac{F\left(n^{\frac{1}{l}} \alpha a+n \alpha^{l} q, \beta b, \gamma c\right)-n^{\frac{1}{l}} \alpha a}{n \alpha^{l}} .
$$

By (3.4) we conclude that $H(0)=q+\frac{1}{l !} a^{l} \frac{\partial^{l} F}{\partial x^{l}}(0,0,0)$. Vesentini's theorem ([13, Theorem 3.4.7]) implies that $\rho_{\mathcal{A}} \circ H$ is a subharmonic function on $\left\{\alpha \in \mathbb{C}: 0<|\alpha|<\frac{1}{\rho(a, b, c)}\right\}$. Moreover, by the maximum principle, we can write $\rho_{\mathcal{A}}(H(0)) \leq \max _{|\alpha|=1} \rho_{\mathcal{A}}(H(\alpha))$. Then Lemma 3.6 of [3] implies that

$$
\begin{aligned}
\rho_{\mathcal{A}}\left(q+\frac{1}{l !} a^{l} \frac{\partial^{l} F}{\partial x^{l}}(0,0,0)\right) & \leq \max _{|\alpha|=1} \rho(H(\alpha))<\frac{1}{n l !} \rho_{\mathcal{A}}\left(a^{l}\right) \rho_{\mathcal{A}}\left(\frac{\partial^{l} F}{\partial x^{l}}(0,0,0)\right) \\
& <\frac{1}{n l !|\alpha|^{l}} \rho_{\mathcal{A}}\left(\frac{\partial^{l} F}{\partial x^{l}}(0,0,0)\right) .
\end{aligned}
$$


The above inequality holds for every $n \geq 1$. Therefore, if $n \rightarrow \infty$, then

$$
\rho_{\mathcal{A}}\left(q+\frac{1}{l !} a^{l} \frac{\partial^{l} F}{\partial x^{l}}(0,0,0)\right)=0
$$

for every $q \in \mathcal{A}$ with $\rho_{\mathcal{A}}(q)=0$. Hence, Theorem 3.4 of [3] implies that $a^{l} \frac{\partial^{l} F}{\partial x^{l}}(0,0,0)$ is in radical of $\mathcal{A}$. Since $\mathcal{A}$ is semi-simple, therefore $a^{l} \frac{\partial^{l} F}{\partial x^{l}}(0,0,0)=0$. Since $a \in \Omega_{\mathcal{A}} \cap Z(\mathcal{A})$, so $a^{l} \neq 0$, and since $\mathcal{A}$ is without of order, therefore $\frac{\partial^{l} F}{\partial x^{l}}(0,0,0)=0$, a contradiction. Thus, our claim is true, and from (3.1), we conclude that $F(a, b, c)=a$. Similarly, we have $F(a, c, b)=a$, $F(b, a, c)=F(b, c, a)=b$ and $F(c, a, b)=F(c, b, a)=c$.

Case (2). In this case, we have $\beta^{l} b^{l} \frac{\partial^{l} F}{\partial y^{l}}(0,0,0) \neq 0$. Again, by (3.1) and (3.3), we have

$$
\begin{aligned}
F\left(\alpha a+n \beta^{l} q, n^{\frac{1}{l}} \beta b, \gamma c\right) & =\alpha a+n \beta^{l} q+\frac{1}{l !} n \beta^{l} b^{l} \frac{\partial^{l} F}{\partial y^{l}}(0,0,0) \\
& =\alpha a+n \beta^{l}\left(q+\frac{1}{l !} b^{l} \frac{\partial^{l} F}{\partial y^{l}}(0,0,0)\right) .
\end{aligned}
$$

Again, by Lemma 2.4 and Lemma 3.6 of [3], we have

$$
\begin{aligned}
\rho\left(\alpha a+n \beta^{l} q, n^{\frac{1}{l}} \beta b, \gamma c\right) & \leq \rho_{\mathcal{A}}\left(\alpha a+n \beta^{l} q\right)+\rho_{\mathcal{A}}\left(n^{\frac{1}{l}} \beta b\right)+\rho_{\mathcal{A}}(\gamma c) \\
& <|\alpha| \rho_{\mathcal{A}}(a)+n^{\frac{1}{l}}|\beta| \rho_{\mathcal{A}}(b)+|\gamma| \rho_{\mathcal{A}}(c) \\
& <\mu\left(\rho_{\mathcal{A}}(a)+\rho_{\mathcal{A}}(b)+\rho_{\mathcal{A}}(c)\right),
\end{aligned}
$$

where $\mu=\max \left\{|\alpha|, n^{\frac{1}{l}}|\beta|,|\gamma|\right\}$. Now, we define a holomorphic function $H$ from $\{\eta \in \mathbb{C}$ : $\left.\mu<\frac{1}{\rho(a, b, c)}, \mu=|\eta|=\max \left\{|\alpha|, n^{\frac{1}{l}}|\beta|,|\gamma|\right\}\right\}$ into $\mathcal{A}$ as follows:

$$
H(\alpha)=\frac{F\left(\alpha a+n \beta^{l} q, n^{\frac{1}{l}} \beta b, \gamma c\right)-\alpha a}{n \beta^{l}} .
$$

Then from (3.7) it follows that $H(0)=q+\frac{1}{l !} b^{l} \frac{\partial^{l} F}{\partial y^{l}}(0,0,0)$. Then $\rho_{\mathcal{A}} \circ H$ is a subharmonic function on $\left\{\eta \in \mathbb{C}: \mu<\frac{1}{\rho(a, b, c)}, \mu=|\eta|=\max \left\{|\alpha|, n^{\frac{1}{l}}|\beta|,|\gamma|\right\}\right\}$. Moreover, Lemma 3.6 of [3] implies that

$$
\begin{aligned}
\rho_{\mathcal{A}}\left(q+\frac{1}{l !} b^{l} \frac{\partial^{l} F}{\partial y^{l}}(0,0,0)\right) & \leq \max _{|\alpha|=1} \rho(H(\alpha)) \\
& <\frac{1}{n l !} \rho_{\mathcal{A}}\left(b^{l}\right) \rho_{\mathcal{A}}\left(\frac{\partial^{l} F}{\partial y^{l}}(0,0,0)\right) \\
& <\frac{1}{n l !|\beta|^{l}} \rho_{\mathcal{A}}\left(\frac{\partial^{l} F}{\partial y^{l}}(0,0,0)\right) .
\end{aligned}
$$

The above inequality holds for every $n \geq 1$. Therefore, if $n \rightarrow \infty$, then

$$
\rho_{\mathcal{A}}\left(q+\frac{1}{l !} b^{l} \frac{\partial^{l} F}{\partial y^{l}}(0,0,0)\right)=0
$$


for every $q \in \mathcal{A}$ with $\rho_{\mathcal{A}}(q)=0$. Hence, Theorem 3.4 of [3] implies that $b^{l} \frac{\partial^{l} F}{\partial y^{l}}(0,0,0)$ is in radical of $\mathcal{A}$. Since $\mathcal{A}$ is semi-simple, therefore $b^{l} \frac{\partial^{l} F}{\partial y^{l}}(0,0,0)=0$. Since $b \in \Omega_{\mathcal{A}} \cap Z(\mathcal{A})$, so $b^{l} \neq 0$. By using that $\mathcal{A}$ is without of order, we conclude that $\frac{\partial^{l} F}{\partial y^{l}}(0,0,0)=0$, a contradiction. Thus, our claim is true, and from (3.1), we conclude that $F(a, b, c)=a$. Similarly, we have $F(a, c, b)=a, F(b, a, c)=F(b, c, a)=b$ and $F(c, a, b)=F(c, b, a)=c$.

Case (3). In this case, we suppose that $i+j=l, i, j \in\{0,1,2,3, \ldots\}$ (without loss of generality, we prove this case for only one $i$ and one $j$ such that $i+j=l$ ). Again by (3.1) and (3.3), we have

$$
\begin{aligned}
F\left(\alpha a+n \alpha^{i} q, n^{\frac{1}{l-i}} \beta b, \gamma c\right) \\
=\alpha a+n \alpha^{i} q+\frac{1}{(l-i) ! i !}\left(\alpha a+n \alpha^{i} q\right)^{i} n \beta^{l-i} b^{l-i} \frac{\partial^{l} F}{\partial x^{i} \partial y^{l-i}}(0,0,0) \\
=\alpha a+n \alpha^{i} q+\frac{1}{(l-i) ! i !}\left(n^{i+1} \alpha^{i^{2}} q^{i} \beta^{l-i} b^{l-i}\right. \\
\left.\quad+i n^{i} \alpha^{i(i-1)+1} q^{i-1} \beta^{l-i} b^{l-i} a+\cdots+n \alpha^{i} a^{i} \beta^{l-i} b^{l-i}\right) \frac{\partial^{l} F}{\partial x^{i} \partial y^{l-i}}(0,0,0) \\
=\alpha a+n \alpha^{i}\left(q+\frac{1}{(l-i) ! i !} a^{i} \beta^{l-i} b^{l-i} \frac{\partial^{l} F}{\partial x^{i} \partial y^{l-i}}(0,0,0)\right) \\
\quad+P(\alpha) \frac{\partial^{l} F}{\partial x^{i} \partial y^{l-i}}(0,0,0) .
\end{aligned}
$$

By Lemma 2.4 and Lemma 3.6 of [3], we have

$$
\begin{aligned}
\rho\left(\alpha a+n \alpha^{i} q, n^{\frac{1}{l-i}} \beta b, \gamma c\right) & \leq \rho_{\mathcal{A}}\left(\alpha a+n \alpha^{i} q\right)+\rho_{\mathcal{A}}\left(n^{\frac{1}{l-i}} \beta b\right)+\rho_{\mathcal{A}}(\gamma c) \\
& <|\alpha| \rho_{\mathcal{A}}(a)+n^{\frac{1}{l-i}}|\beta| \rho_{\mathcal{A}}(b)+|\gamma| \rho_{\mathcal{A}}(c) \\
& <\mu\left(\rho_{\mathcal{A}}(a)+\rho_{\mathcal{A}}(b)+\rho_{\mathcal{A}}(c)\right),
\end{aligned}
$$

where $\mu=\max \left\{|\alpha|, n^{\frac{1}{l-i}}|\beta|,|\gamma|\right\}$. Now, we define a holomorphic function $H$ from $\{\eta \in \mathbb{C}$ : $\left.\mu<\frac{1}{\rho(a, b, c)}, \mu=|\eta|=\max \left\{|\alpha|, n^{\frac{1}{l-i}}|\beta|,|\gamma|\right\}\right\}$ into $\mathcal{A}$ as follows:

$$
H(\lambda)=\frac{F\left(\alpha a+n \alpha^{i} q, n^{\frac{1}{l-i}} \beta b, \gamma c\right)-\alpha a}{n \alpha^{i}} .
$$

Then from (3.7) it follows that $H(0)=q+\frac{1}{(l-i) ! ! !} a^{i} \beta^{l-i} b^{l-i} \frac{\partial^{l} F}{\partial x^{i} \partial y^{l-i}}(0,0,0)$. Then $\rho_{\mathcal{A}} \circ H$ is a subharmonic function on $\left\{\eta \in \mathbb{C}: \mu<\frac{1}{\rho(a, b, c)}, \mu=|\eta|=\max \left\{|\alpha|, n^{\frac{1}{l-i}}|\beta|,|\gamma|\right\}\right\}$. Moreover, Lemma 3.6 of [3] implies that

$$
\begin{aligned}
& \rho_{\mathcal{A}}\left(q+\frac{1}{(l-i) ! i !} a^{i} \beta^{l-i} b^{l-i} \frac{\partial^{l} F}{\partial x^{i} \partial y^{l-i}}(0,0,0)\right) \\
& \quad \leq \max _{|\alpha|=1} \rho(H(\alpha)) \\
& \quad<\frac{|\beta|^{l-i}}{n(l-i) ! i !} \rho_{\mathcal{A}}\left(a^{i}\right) \rho_{\mathcal{A}}\left(b^{l-i}\right) \rho_{\mathcal{A}}\left(\frac{\partial^{l} F}{\partial x^{i} \partial y^{l-i}}(0,0,0)\right) \\
& \quad<\frac{1}{n(l-i) ! i !|\alpha|^{i}} \rho_{\mathcal{A}}\left(\frac{\partial^{l} F}{\partial x^{i} \partial y^{l-i}}(0,0,0)\right) .
\end{aligned}
$$


The above inequality holds for every $n \geq 1$. Therefore, if $n \longrightarrow \infty$, then

$$
\rho_{\mathcal{A}}\left(q+\frac{1}{(l-i) ! i !} a^{i} \beta^{l-i} b^{l-i} \frac{\partial^{l} F}{\partial x^{i} \partial y^{l-i}}(0,0,0)\right)=0
$$

for every $q \in \mathcal{A}$ with $\rho_{\mathcal{A}}(q)=0$. Hence, $a^{i} \beta^{l-i} b^{l-i} \frac{\partial^{l} F}{\partial x^{i} \partial y^{l-i}}(0,0,0)$ is in radical of $\mathcal{A}$, therefore $a^{i} \beta^{l-i} b^{l-i} \frac{\partial^{l} F}{\partial x^{i} \partial y^{l-i}}(0,0,0)=0$. Since $\beta^{l-i} \neq 0$ and $a, b \in \Omega_{\mathcal{A}} \cap Z(\mathcal{A})$, so $a^{i} \neq 0$ and $b^{l-i} \neq 0$. Again, by using that $\mathcal{A}$ is without of order, we conclude that $\frac{\partial^{l} F}{\partial x^{i} \partial y^{l-i}}(0,0,0)=0$, a contradiction. Thus, our claim is true, and from (3.1), we conclude that $F(a, b, c)=a$. Similarly, we have $F(a, c, b)=a, F(b, a, c)=F(b, c, a)=b$ and $F(c, a, b)=F(c, b, a)=c$.

Case (4). Let $1 \leq i+j \leq l$. Then we have $\alpha^{i} a^{i} \beta^{j} b^{j} \gamma^{l-i-j} c^{l-i-j} \frac{\partial^{l} F}{\partial x^{i} \partial y^{j} \partial z^{l-i-j}}(0,0,0) \neq 0$. Again, by (3.1) and (3.3), we have

$$
\begin{aligned}
& F\left(\alpha a+n \alpha^{i} q, \beta b, n^{\frac{1}{l-i-j}} \gamma c\right) \\
& =\alpha a+n \alpha^{i} q+\frac{1}{i ! j !(l-i-j) !}\left(\left(\alpha a+n \alpha^{i} q\right)^{i}(\beta b)^{j}\left(n^{\frac{1}{l-i-j}} \gamma c\right)^{l-i-j} \frac{\partial^{l} F}{\partial x^{i} \partial y^{j} \partial z^{l-i-j}}(0,0,0)\right) \\
& =\alpha a+n \alpha^{i} q+\frac{1}{i ! j !(l-i-j) !}\left(n^{i+1} \alpha^{i^{2}} q^{i} \beta^{j} b^{j} \gamma^{l-i-j} c^{l-i-j}+i a n^{i} \alpha^{i(i-1) i+1} q^{i-1} \beta^{j} b^{j} \gamma^{l-i-j} c^{l-i-j}\right. \\
& \left.\quad+\cdots+n \alpha^{i} a^{i} \beta^{j} b^{j} \gamma^{l-i-j} c^{l-i-j}\right) \frac{\partial^{l} F}{\partial x^{i} \partial y^{j} \partial z^{l-i-j}}(0,0,0) \\
& =\alpha a+n \alpha^{i}\left(q+\frac{1}{i ! j !(l-i-j) !} a^{i} \beta^{j} b^{j} \gamma^{l-i-j} c^{l-i-j} \frac{\partial^{l} F}{\partial x^{i} \partial y^{j} \partial z^{l-i-j}}(0,0,0)\right) \\
& \quad+P(\alpha) \frac{\partial^{l} F}{\partial x^{i} \partial y^{j} \partial z^{l-i-j}}(0,0,0) .
\end{aligned}
$$

Then

$$
\begin{aligned}
\rho\left(\alpha a+n \alpha^{i} q, \beta b, n^{\frac{1}{l-i-j}} \gamma c\right) & \leq \rho_{\mathcal{A}}\left(\alpha a+n \alpha^{i} q\right)+\rho_{\mathcal{A}}(\beta b)+\rho_{\mathcal{A}}\left(n^{\frac{1}{l-i-j}} \gamma c\right) \\
& <|\alpha| \rho_{\mathcal{A}}(a)+|\beta| \rho_{\mathcal{A}}(b)+n^{\frac{1}{l-i-j}}|\gamma| \rho_{\mathcal{A}}(c) \\
& <\mu\left(\rho_{\mathcal{A}}(a)+\rho_{\mathcal{A}}(b)+\rho_{\mathcal{A}}(c)\right),
\end{aligned}
$$

where $\mu=\max \left\{|\alpha|,|\beta|, n^{\frac{1}{l-i-j}}|\gamma|\right\}$. Now, we define a holomorphic function $H$ from $\{\eta \in \mathbb{C}$ : $\left.\mu<\frac{1}{\rho(a, b, c)}, \mu=|\eta|=\max \left\{|\alpha|,|\beta|, n^{\frac{1}{l-i-j}}|\gamma|\right\}\right\}$ into $\mathcal{A}$ as follows:

$$
H(\alpha)=\frac{F\left(\alpha a+n \alpha^{i} q, \beta b, n^{\frac{1}{l-i-j}} \gamma c\right)-\alpha a}{n \alpha^{i}} .
$$

Then from (3.9) it follows that $H(0)=q+\frac{1}{i \cdot j !(l-i-j) !}\left(a^{i} \beta^{j} b^{j} \gamma^{l-i-j} c^{l-i-j} \frac{\partial^{l} F}{\partial x^{i} \partial y^{j} \partial z^{l-i-j}}(0,0,0)\right)$. Then $\rho_{\mathcal{A}} \circ H$ is a subharmonic function on $\left\{\eta \in \mathbb{C}: \mu<\frac{1}{\rho(a, b, c)}, \mu=|\eta|=\max \{|\alpha|,|\beta|\right.$, $\left.\left.n^{\frac{1}{l-i-j}}|\gamma|\right\}\right\}$. Therefore,

$$
\begin{aligned}
& \rho_{\mathcal{A}}\left(q+\frac{1}{i ! j !(l-i-j) !} a^{i} \beta^{j} b^{j} \gamma^{l-i-j} c^{l-i-j} \frac{\partial^{l} F}{\partial x^{i} \partial y^{j} \partial z^{l-i-j}}(0,0,0)\right) \\
& \quad \leq \max _{|\alpha|=1} \rho(H(\alpha))
\end{aligned}
$$




$$
\begin{aligned}
& <\frac{|\beta|^{j}|\gamma|^{l-i-j}}{n i ! j !(l-i-j) !} \rho_{\mathcal{A}}\left(a^{i}\right) \rho_{\mathcal{A}}\left(b^{j}\right) \rho_{\mathcal{A}}\left(c^{l-i-j}\right) \rho_{\mathcal{A}}\left(\frac{\partial^{l} F}{\partial x^{i} \partial y^{j} \partial z^{l-i-j}}(0,0,0)\right) \\
& <\frac{1}{n i j \cdot j !(l-i-j) !|\alpha|^{i}} \rho_{\mathcal{A}}\left(\frac{\partial^{l} F}{\partial x^{i} \partial y^{j} \partial z^{l-i-j}}(0,0,0)\right) .
\end{aligned}
$$

Therefore, if $n \longrightarrow \infty$, then

$$
\rho_{\mathcal{A}}\left(q+\frac{1}{i ! j !(l-i-j) !} a^{i} \beta^{j} b^{j} \gamma^{l-i-j} c^{l-i-j} \frac{\partial^{l} F}{\partial x^{i} \partial y^{j} \partial z^{l-i-j}}(0,0,0)\right)=0
$$

for every $q \in \mathcal{A}$ with $\rho_{\mathcal{A}}(q)=0$. Hence, $a^{i} \beta^{j} b^{j} \gamma^{l-i-j} c^{l-i-j} \frac{\partial^{l} F}{\partial x^{i} \partial y^{j} \partial z^{l-i-j}}(0,0,0)$ is in radical of $\mathcal{A}$. Since $\mathcal{A}$ is semi-simple, therefore $a^{i} \beta^{j} b^{j} \gamma^{l-i-j} c^{l-i-j} \frac{\partial^{l} F}{\partial x^{i} \partial y^{j} j z^{l-i-j}}(0,0,0)=0$. Since $\beta^{j} \neq 0, \gamma^{l-i-j} \neq 0$ and $a, b \in \Omega_{\mathcal{A}} \cap Z(\mathcal{A})$, so $a^{i} \neq 0, b^{j}$ and $c^{l-i-j} \neq 0$, we conclude that $\frac{\partial^{l} F}{\partial x^{i} \partial y^{j} j z^{l-i-j}}(0,0,0)=0$, a contradiction. Thus, our claim is true, and from (3.1), we conclude that $F(a, b, c)=a$. Similarly, we have $F(a, c, b)=a, F(b, a, c)=F(b, c, a)=b$ and $F(c, a, b)=F(c, b, a)=c$.

Case (5). Now, let $i=j=0$. Then we have $\gamma^{l} c^{l} \frac{\partial^{l} F}{\partial z^{l}}(0,0,0) \neq 0$. Similar to the previous cases, we have

$$
\begin{aligned}
F\left(\alpha a+n \gamma^{l} q, \beta b, n^{\frac{1}{l}} \gamma c\right) & =\alpha a+n \gamma^{l} q+\frac{1}{l !} n \gamma^{l} c^{l} \frac{\partial^{l} F}{\partial z^{l}}(0,0,0) \\
& =\alpha a+n \gamma^{l}\left(q+\frac{1}{l !} c^{l^{l} F} \frac{\partial^{l}}{\partial z^{l}}(0,0,0)\right) .
\end{aligned}
$$

Then

$$
\begin{aligned}
\rho\left(\alpha a+n \gamma^{l} q, \beta b, n^{\frac{1}{l}} \gamma c\right) & \leq \rho_{\mathcal{A}}\left(\alpha a+n \gamma^{l} q\right)+\rho_{\mathcal{A}}(\beta b)+\rho_{\mathcal{A}}\left(n^{\frac{1}{l}} \gamma c\right) \\
& <|\alpha| \rho_{\mathcal{A}}(a)+|\beta| \rho_{\mathcal{A}}(b)+n^{\frac{1}{l}}|\gamma| \rho_{\mathcal{A}}(c) \\
& <\mu\left(\rho_{\mathcal{A}}(a)+\rho_{\mathcal{A}}(b)+\rho_{\mathcal{A}}(c)\right),
\end{aligned}
$$

where $\mu=\max \left\{|\alpha|,|\beta|, n^{\frac{1}{l}}|\gamma|\right\}$. Now, we define a holomorphic function $H$ from $\{\eta \in \mathbb{C}$ : $\left.\mu<\frac{1}{\rho(a, b, c)}, \mu=|\eta|=\max \left\{|\alpha|,|\beta|, n^{\frac{1}{l}}|\gamma|\right\}\right\}$ into $\mathcal{A}$ as follows:

$$
H(\alpha)=\frac{F\left(\alpha a+n \gamma^{l} q, \beta b, n^{\frac{1}{l}} \gamma c\right)-\alpha a}{n \gamma^{l}} .
$$

Then from (3.11) it follows that $H(0)=q+\frac{1}{l !} c \frac{\partial}{\partial z^{l}}(0,0,0)$. Then $\rho_{\mathcal{A}} \circ H$ is a subharmonic function on $\left\{\eta \in \mathbb{C}: \mu<\frac{1}{\rho(a, b, c)}, \mu=|\eta|=\max \left\{|\alpha|,|\beta|, n^{\frac{1}{l}}|\gamma|\right\}\right\}$, and

$$
\begin{aligned}
\rho_{\mathcal{A}}\left(q+\frac{1}{l !} c^{l} \frac{\partial^{l} F}{\partial z^{l}}(0,0,0)\right) & \leq \max _{|\alpha|=1} \rho(H(\alpha)) \\
& <\frac{1}{n l !} \rho_{\mathcal{A}}\left(c^{l}\right) \rho_{\mathcal{A}}\left(\frac{\partial^{l} F}{\partial z^{l}}(0,0,0)\right) \\
& <\frac{1}{n l !|\gamma|^{l}} \rho_{\mathcal{A}}\left(\frac{\partial^{l} F}{\partial z^{l}}(0,0,0)\right) .
\end{aligned}
$$


Therefore, if $n \longrightarrow \infty$, then

$$
\rho_{\mathcal{A}}\left(q+\frac{1}{l !} c^{l} \frac{\partial^{l} F}{\partial z^{l}}(0,0,0)\right)=0
$$

for every $q \in \mathcal{A}$ with $\rho_{\mathcal{A}}(q)=0$. Hence, $c^{l} \frac{\partial^{l} F}{\partial z^{l}}(0,0,0)$ is in radical of $\mathcal{A}$. Therefore, $c^{l} \frac{\partial^{l} F}{\partial z^{l}}(0,0,0)=0$. Since $c \in \Omega_{\mathcal{A}} \cap Z(\mathcal{A})$, so $c^{l} \neq 0$, then $\frac{\partial^{l} F}{\partial z^{l}}(0,0,0)=0$, a contradiction. Thus, (3.1) implies that our claim is true, and from (3.1), we conclude that $F(a, b, c)=a$. Similarly, we have $F(a, c, b)=a, F(b, a, c)=F(b, c, a)=b$ and $F(c, a, b)=F(c, b, a)=c$.

By gathering the above five cases, we conclude $(a, b, c)$ is a tripled fixed point for $F$, and since $(a, b, c)$ was arbitrary, so every point of $\Omega_{\mathcal{A} \times \mathcal{A} \times \mathcal{A}} \cap Z(\mathcal{A} \times \mathcal{A} \times \mathcal{A})$ is a tripled fixed point for $F$.

Corollary 3.2 Let $\mathcal{A}$ be a unital without of order semi-simple Banach algebra. If $F$ : $\Omega_{\mathcal{A} \times \mathcal{A} \times \mathcal{A}} \subseteq \mathcal{A} \times \mathcal{A} \times \mathcal{A} \longrightarrow \Omega_{\mathcal{A}}$ is a holomorphic map that satisfies the conditions $F(0,0,0)=0, \frac{\partial F}{\partial x}(0,0,0)=\operatorname{id}_{\mathcal{A}}, \frac{\partial F}{\partial y}(0,0,0)=0, \frac{\partial F}{\partial z}(0,0,0)=0, \frac{\partial^{2} F}{\partial x^{i} \partial y^{j} \partial z^{k}}(0,0,0)=0$, where $i+j+k=2, i, j, k=0,1,2$, and $\frac{\partial^{3} F}{\partial x^{i} \partial y^{j} \partial z^{k}}(0,0,0)=0$, where $i+j+k=3, i, j, k=0,1,2,3$, then every $(a, b, c) \in \Omega_{\mathcal{A} \times \mathcal{A} \times \mathcal{A}} \cap Z(\mathcal{A} \times \mathcal{A} \times \mathcal{A})$ is a tripled fixed point for $F$.

In the following theorem, we characterize tripled fixed points of holomorphic functions on FLM algebras.

Theorem 3.3 Let $\mathcal{A}$ be a unital without of order complete semi-simple metrizable FLM algebra. For given $(a, b, c) \in \Omega_{\mathcal{A} \times \mathcal{A} \times \mathcal{A}} \backslash Z(\mathcal{A} \times \mathcal{A} \times \mathcal{A})$, there is a holomorphic map $F$ : $\Omega_{\mathcal{A} \times \mathcal{A} \times \mathcal{A}} \longrightarrow \Omega_{\mathcal{A}}$ satisfying the conditions $F(0,0,0)=0, \frac{\partial F}{\partial x}(0,0,0)=\mathrm{id}_{\mathcal{A}}, \frac{\partial F}{\partial y}(0,0,0)=0$, $\frac{\partial F}{\partial z}(0,0,0)=0, \frac{\partial^{2} F}{\partial x^{i} \partial y^{j} \partial z^{k}}(0,0,0)=0$, where $i+j+k=2, i, j, k=0,1,2$, and $\frac{\partial^{3} F}{\partial x^{i} \partial y^{j} \partial z^{k}}(0,0,0)=0$, where $i+j+k=3, i, j, k=0,1,2,3$, such that $F(a, b, c) \neq a, F(b, a, c) \neq b$ and $F(c, a, b) \neq c$.

Proof Let $(a, b, c) \in \Omega_{\mathcal{A} \times \mathcal{A} \times \mathcal{A}} \backslash Z(\mathcal{A} \times \mathcal{A} \times \mathcal{A})$. Then there exist $(u, u, u) \in \mathcal{A} \times \mathcal{A} \times \mathcal{A}$ such that

$$
(u a, u b, u c) \neq(a u, b u, c u) \text {. }
$$

Let $D_{\mathcal{A} \times \mathcal{A} \times \mathcal{A}}(u, u, u)<1$, then $D_{\mathcal{A}}(u)<1$. Define $U:=\log (e-u)$, then

$$
e^{-U} a e^{U} \neq a, \quad e^{-U} b e^{U} \neq b \quad \text { and } \quad e^{-U} c e^{U} \neq c .
$$

Now, define $F: \Omega_{\mathcal{A} \times \mathcal{A} \times \mathcal{A}} \longrightarrow \Omega_{\mathcal{A}}$ as follows:

$$
F(x, y, z)=e^{-\frac{x^{2} z^{2} u}{a^{2} c^{2}}} x e^{\frac{y^{2} z^{2} u}{b^{2} c^{2}}}
$$

for every $(x, y, z)$ in $\Omega_{\mathcal{A} \times \mathcal{A} \times \mathcal{A}}$. Clearly, $F$ is a holomorphic function, $F(0,0,0)=0$, $\frac{\partial F}{\partial x}(0,0,0)=\operatorname{id}_{\mathcal{A}}, \frac{\partial F}{\partial y}(0,0,0)=0, \frac{\partial F}{\partial z}(0,0,0)=0, \frac{\partial^{2} F}{\partial x^{i} \partial y^{j} \partial z^{k}}(0,0,0)=0$, where $i+j+k=2$, $i, j, k=0,1,2$, and $\frac{\partial^{3} F}{\partial x^{i} \partial y^{j} \partial z^{k}}(0,0,0)=0$, where $i+j+k=3, i, j, k=0,1,2,3$, but $F(a, b, c) \neq a$, and similarly, we can show that there is a holomorphic map $F: \Omega_{\mathcal{A} \times \mathcal{A} \times \mathcal{A}} \longrightarrow \Omega_{\mathcal{A}}$ with the required conditions such that $F(b, a, c) \neq b$ and $F(c, a, b) \neq c$. 
Example 3.4 Let $X=\mathbb{R}$ be the space of real numbers and let $F: X \times X \rightarrow X$ be a function defined by $F(x, y, z)=x$ that satisfies the conditions of Theorem 3.1.

Example 3.5 Let $X$ be a unital without of order complete semi-simple Banach algebra and let $F: X \times X \rightarrow X$ be a function defined by $F(x, y, z)=e^{y^{2} z^{2}} x e^{-y^{2} z^{2}}$ that satisfies the conditions of Theorem 3.1. For example, let $X=M(G)$ be the measure space on a locally compact Hausdorff space $G$. Another algebra that we can choose is $\ell^{1}(G)$, where $G$ is a locally compact discrete group.

Corollary 3.6 Let $\mathcal{A}$ be a unital without of order semi-simple Banach algebra. For given $(a, b, c) \in \Omega_{\mathcal{A} \times \mathcal{A} \times \mathcal{A}} \backslash Z(\mathcal{A} \times \mathcal{A} \times \mathcal{A})$, there is a holomorphic map $F: \Omega_{\mathcal{A} \times \mathcal{A} \times \mathcal{A}} \rightarrow \Omega_{\mathcal{A}}$ satisfying the conditions $F(0,0,0)=0, \frac{\partial F}{\partial x}(0,0,0)=\mathrm{id}_{\mathcal{A}}, \frac{\partial F}{\partial y}(0,0,0)=0, \frac{\partial F}{\partial z}(0,0,0)=0$, $\frac{\partial^{2} F}{\partial x^{i} \partial y^{j} \partial z^{k}}(0,0,0)=0$, where $i+j+k=2, i, j, k=0,1,2$, and $\frac{\partial^{3} F}{\partial x^{i} \partial y^{j} \partial z^{k}}(0,0,0)=0$, where $i+j+k=3, i, j, k=0,1,2,3$, such that $F(a, b, c) \neq a, F(b, a, c) \neq b$ and $F(c, a, b) \neq c$.

\section{Competing interests}

The authors declare that they have no competing interests.

\section{Authors' contributions}

All authors contributed equally and significantly in writing this paper. All authors read and approved the final manuscript.

Received: 4 July 2012 Accepted: 9 January 2013 Published: 25 January 2013

\section{References}

1. Ansari-Piri, E: A class of factorable topological algebras. Proc. Edinb. Math. Soc. 33, 53-59 (1990)

2. Ansari-Piri, E: Topics on fundamental topological algebras. Honam Math. J. 23, 59-66 (2001)

3. Zohri, A, Jabbari, A: Generalization of some properties of Banach algebras to fundamental locally multiplicative topological algebras. Turk. J. Math. 36, 445-451 (2012)

4. Gnana Bhaskar, T, Lakshmikantham, V: Fixed point theorems in partially ordered metric spaces and applications. Nonlinear Anal. 65, 1379-1393 (2006)

5. Samet, B, Vetro, C: Coupled fixed point, $f$-invariant set and fixed point of N-order. Ann. Funct. Anal. 1(2), 46-56 (2010)

6. Abbas, M, Sintunavarat, W, Kumam, P: Coupled fixed point of generalized contractive mappings on partially ordered G-metric spaces. Fixed Point Theory Appl. 2012, 31 (2012)

7. Sintunavarat, W, Cho, YJ, Kumam, P: Coupled coincidence point theorems for contractions without commutative condition in intuitionistic fuzzy normed spaces. Fixed Point Theory Appl. 2011, 81 (2011)

8. Sintunavarat, W, Cho, YJ, Kumam, P: Coupled fixed point theorems for weak contraction mapping under F-invariant set. Abstr. Appl. Anal. 2012, Article ID 324874 (2012)

9. Sintunavarat, W, Cho, YJ, Kumam, P: Coupled fixed point theorems for contraction mapping induced by cone ball-metric in partially ordered spaces. Fixed Point Theory Appl. 2012, 128 (2012)

10. Berinde, $V$, Borcut, M: Tripled fixed point theorems for contractive type mappings in partially ordered metric spaces. Nonlinear Anal. 74(15), 4889-4897 (2011)

11. Aydi, $H$, Abbas, $M$, Sintunavarat, $W$, Kumam, $P$ : Tripled fixed point of $W$-compatible mappings in abstract metric spaces. Fixed Point Theory Appl. 2012, 134 (2012)

12. Ansari-Piri, E: The linear functionals on fundamental locally multiplicative topological algebras. Turk. J. Math. 34, 385-391 (2010)

13. Aupetit, B: A Primer on Spectral Theory. Springer, New York (1991)

doi:10.1186/1687-1812-2013-16

Cite this article as: Razani and Hosseinzadeh: Triple fixed point theorems on FLM algebras. Fixed Point Theory and Applications 2013 2013:16. 